\title{
An outbreak of Shiga toxin-producing Escherichia coli O157: H7 associated with contaminated salad leaves: epidemiological, genomic and food trace back investigations
}

\author{
A. F. W. MIKHAIL ${ }^{1}$, C. JENKINS ${ }^{1}$ *, T. J. DALLMAN ${ }^{1}$, T. INNS $^{2,3}$, A. DOUGLAS ${ }^{1}$, \\ A. I. C. MARTÍN ${ }^{4}$, A. FOX ${ }^{1}$, P. CLEARY ${ }^{2,3}$, R. ELSON $^{1}$ AND J. HAWKER ${ }^{2,3}$ \\ ${ }^{1}$ National Infection Service, Public Health England, London, UK \\ ${ }^{2}$ Field Epidemiology Services, Public Health England, London, UK \\ ${ }^{3}$ NIHR Health Protection Research Unit in Gastrointestinal Infections, London, UK \\ ${ }^{4}$ European Program for Intervention Epidemiology Training (EPIET), European Centre for Disease Prevention \\ and Control, (ECDC), Stockholm, Sweden
}

Received 2 August 2017; Final revision 18 November 2017; Accepted 21 November 2017; first published online 18 December 2017

\section{SUMMARY}

In August 2015, Public Health England detected an outbreak of Shiga toxin-producing Escherichia coli (STEC) serotype O157:H7 caused by contaminated salad leaves in a mixed leaf prepacked salad product from a national retailer. The implicated leaves were cultivated at five different farms and the zoonotic source of the outbreak strain was not determined. In March 2016, additional isolates from new cases were identified that shared a recent common ancestor with the outbreak strain. A case-case study involving the cases identified in 2016 revealed that ovine exposures were associated with illness ( $n=16$; AOR 8.24; 95\% CI 1.55-39·74). By mapping the recent movement of sheep and lambs across the United Kingdom, epidemiological links were established between the cases reporting ovine exposures. Given the close phylogenetic relationship between the outbreak strain and the isolates from cases with ovine exposures, it is plausible that ovine faeces may have contaminated the salad leaves via untreated irrigation water or run-off from fields nearby. Timely and targeted veterinary and environmental sampling should be considered during foodborne outbreaks of STEC, particularly where ready to eat vegetables and salads are implicated.

Key words: Bacterial typing, epidemiology, public health microbiology, Shiga-like toxin-producing E. coli, zoonotic foodborne diseases.

\section{INTRODUCTION}

Shiga toxin-producing Escherichia coli (STEC) serotype O157:H7 emerged as a significant risk to public health in the 1980s, associated with outbreaks of gastrointestinal symptoms and haemolytic uraemic syndrome (HUS) [1]. Microbiological and epidemiological

\footnotetext{
* Author for correspondence: Dr Claire Jenkins, Gastrointestinal Bacteria Reference Unit, Public Health England, 61 Colindale Avenue, London NW9 5EQ, UK.

(Email: claire.jenkins@phe.gov.uk)
}

investigations identified ruminants (mainly cattle and sheep) as the zoonotic reservoir [2]. Transmission occurs by consumption of contaminated food or water, or direct contact with animals or their environment [3]. The infectious dose is low (10-100 organisms) and person-to-person spread can occur in households, nurseries and other institutional settings.

Prior to 2005, outbreaks in England were often linked to the consumption of contaminated undercooked beef or cross-contamination of cooked meats [1]. Since that time, reports of meat-related outbreaks

\footnotetext{
$\$$ The original version of this article was submitted without one of the authors credited. A notice detailing this has been published and the error rectified in the online PDF and HTML copies.
} 
have decreased and outbreaks associated with eating contaminated raw vegetables and salad leaves have been reported more regularly [4-6]. The largest national outbreak in the United Kingdom (252 cases) was caused by STEC O157:H7 PT8 occurred in 2011 and was linked to handling raw leeks and potatoes [7].

At Public Health England (PHE), whole genome sequencing (WGS) is used for the routine surveillance and detection of outbreaks caused by STEC O157:H7. WGS data have facilitated a more robust approach to case ascertainment and provided insight into the investigation of complex outbreaks scenarios [8-10]. In August 2015, an outbreak of STEC O157:H7 phage type (PT) 8 was detected following routine monitoring of the WGS typing data. The aim of this study was to identify the original source and likely transmission routes associated with this outbreak.

\section{METHODS}

\section{Microbiology}

In England and Wales, all presumptive isolates of STEC 0157:H7 from clinical cases detected at local hospital laboratories, and isolates from food, animal and environmental samples detected at commercial or government-funded laboratories, are referred to the Gastrointestinal Bacteria Reference Unit (GBRU) at PHE for confirmation and further typing. Isolates are confirmed as STEC O157 using PCR targeting stxl, st $x 2$, eae encoding the adhesin, intimin and $0157 \mathrm{rfb}$ which is part of the $\mathrm{O} 157$ antigen encoding cluster. All isolates submitted during the period of this study were phage typed and genome sequenced. Genes encoding the $\mathrm{H} 7$ flagella antigen were detected in the genome sequence, as previously described [11].

For WGS, DNA was extracted from cultures of STEC O157:H7 for sequencing on the Illumina HiSeq 2500 instrument. High-quality Illumina reads were mapped to the STEC O157:H7 reference genome Sakai (Genbank accession BA000007) using BWA-MEM [12]. Single nucleotide polymorphisms (SNPs) were identified using GATK2 [13] in unified genotyper mode. Core genome positions that had a high-quality SNP $(>90 \%$ consensus, minimum depth $10 \times, G Q \geqslant 30$ ) in at least one isolate were extracted and RaxML [14] was used to derive the maximum likelihood phylogeny of the isolates. Isolates with $<30 \times$ coverage when mapping to the O157:H7 reference genome were discarded from analysis. Positions with $<10 \times$ coverage or where the consensus base was present in $<90 \%$ of the reads were discarded from analysis. The average coverage obtained in the study was $55 \cdot 5 \times$.

Genomes were compared to the sequences held in the PHE STEC O157:H7 WGS database. Isolates of STEC O157:H7 with less than five SNPs differences within their core genome are considered closely related and likely to have an epidemiological link [15]. Hierarchical single linkage clustering was performed on the pairwise SNP difference between all isolates at various distance thresholds $(\Delta 250, \Delta 100, \Delta 50, \Delta 25$, $\Delta 10, \Delta 5, \Delta 0)$ [16]. The result of the clustering is a SNP address that can be used to describe the population structure based on clonal groups. Although isolates greater than 5 SNPs apart are unlikely to be part of the same temporally linked outbreak, deeper phylogenetic relationships within the 10 or 25 SNP clusters may provide epidemiologically useful information or associations [15]. Stx subtyping was performed as described by Ashton et al. [17].

Timed phylogenies were constructed using BEASTMCMC v1.80, as previously described [15]. A relaxed log normal clock rate under a constant population size was selected as found to be optimal for STEC O157:H7 in our previous study [18]. The model was run with a chain length of one billion and a maximum clade credibility tree was constructed using TreeAnnotator v1.75.

FASTQ reads from all sequences in this study and the PHE STEC O157:H7 WGS data can be found at the PHE Pathogens BioProject at the National Center for Biotechnology Information (Accession PRJNA248792) (Supplementary Table S1).

\section{Epidemiological investigations}

Presumptive cases of STEC are reported directly to PHE centres by clinical microbiologists at local hospital laboratories. A standardised STEC Enhanced Surveillance Questionnaire (SESQ) (https://www.gov. uk/government/uploads/system/uploads/attachment_ data/file/323423/VTEC_Questionnaire.pdf) is administered to cases either by local health protection professionals or environmental health practitioners (EHPs). Data from the questionnaires are entered into the National Enhanced Surveillance System for STEC in England (NESSS) [1].

\section{Case-case investigations}

Based in the initial analysis of the SESQ data, a casecase study design was used to test the hypothesis that 
outbreak cases were associated with prepacked salad from the national retailer (retailer X). The analysis was conducted in Stata 13.0. Existing STEC enhanced surveillance questionnaires were used for both outbreak cases and control cases. Only responses to binary questions were included in the analysis. Outbreak cases were defined as primary symptomatic cases of STEC 0157:H7 PT8 stx2a, belonging to the same five SNP single linkage cluster (hereafter referred to as the outbreak strain), with the SNP address $18 \cdot 35 \cdot 380 \cdot 765 \cdot 1009 \cdot 1526 . \%$ confirmed by GBRU with onset of diarrhoea between 19 July and 30 September 2015, and resident in England or Wales. Only the first 45 outbreak cases were included in this analysis, as the remaining three cases were identified after the case-case study had been completed. For each outbreak case, two control cases were selected from indigenous cases of STEC O157:H7 with PTs other than PT8 associated with illness in July and August reported to the national surveillance system during the preceding years (2010-2014). Outbreak cases and control cases were not matched on geography since cases were distributed across England. Odds ratios were calculated for each single binary exposure with a $\chi^{2}$ test; multivariable logistic regression was then performed using a forward-backwards approach: first, exposures with a $P$ value $<0.05$ were included and a backwards stepwise procedure undertaken. Exposures found to be significant or potential confounders were kept. The rest of the exposures $(P$ value $0 \cdot 05-0 \cdot 2)$ were added to a second model and the same backwards stepwise procedure undertaken. An alternative model was also constructed in which control cases were restricted to those with onset in July and August 2015, to account for a change in the market share of retailer $\mathrm{X}$ in the preceding year.

A second case-case study was conducted to test the hypothesis that illness in cases detected after the outbreak, with isolates within 10 SNPs of the outbreak strain, was associated with consumption of lamb meat and/or exposure to live sheep or lambs. The analyses were conducted with the statistical software package R version 3.3.2 (http://www.R-project.org). Cluster cases were defined as primary or co-primary with isolates belonging to the $10 \mathrm{SNP}$ single linkage cluster designated $18 \cdot 35 \cdot 380 \cdot 765 \cdot 1009 . \%$, and onset between 1 January and 31 December 2016. Indigenous cases of STEC O157:H7 infection reported to the national surveillance system from 2009 to 2016 inclusive with PTs other than PT8, frequency-matched to cases in terms of month of onset and PHE centre of residence, were used as control cases. In this analysis, details from free text responses were also included and parsed into single word binary variables with natural language processing techniques (http://www.jstatsoft. org/v25/i05/) [19]. A high ratio of control cases to cluster cases was used $(120: 1 ; 1919$ controls, 16 cases) to facilitate analysis of free text-derived variables with low representation. Odds ratios were calculated with Fisher's exact test. Multivariable logistic regression was conducted on exposures from the single variable analysis that were experienced by at least $20 \%$ of cases and had an odds ratio and lower $95 \%$ confidence interval $>1$. Age and sex were also included in the model a priori. A forward-backwards approach was used to derive the final model, as described in the previous section.

\section{Microbiological examination of food and environmental samples}

Food, water and environmental samples were collected by EHPs from the retailer $(n=68)$, distributor $(n=24)$ and the five growers implicated in the supply chain $(n$ =55) (Supplementary Table S2), and transported in accordance with the Food Standards Agency Food Law Code of Practice (https://www.food.gov.uk/ enforcement/codes-of-practice/food-law-code-of-practice-2015) to PHE Food, Water and Environmental Microbiology Laboratories at Preston, Porton, London, York or Birmingham in cold boxes at a temperature of between 0 and $8{ }^{\circ} \mathrm{C}$ and tested within $24 \mathrm{~h}$ of collection. Testing of the food samples followed PHE Standard Method F17 based on BS EN ISO 16654:2001 http:// img.21 food.cn/img/biaozhun/20100729/181/11294219. pdf, as previously described [8].

\section{Investigation of animal movements with the network software FoodChain-Lab}

The locations and details of farms and lairage where lambs associated with the contaminated meat products were kept immediately prior to slaughter, and farms where cases had reported exposure to live sheep in the 7 days preceding the onset of symptoms were obtained and recorded. Data on the movement of sheep and lambs to and from these sites were requested from the Animal Reporting \& Movement Service (ARAMS) (http://www.arams.co.uk/), a system which records the movements of sheep, goat and deer between agricultural premises, livestock markets and slaughterhouses in the UK. All movement data 
between May 2015 and October 2016 were requested. The locations and details of farms located most closely to the salad growers associated with the 2015 outbreak were also obtained and recorded but data for sheep and lamb movement onto and off these sites were not available for all requests for information.

Using the open source software, FoodChain-Lab (http://silebat.github.io/BfROpenLab/fcl_home.html), a network of sheep and lamb movements was constructed. FoodChain-Lab facilitates the identification of possible outbreak sources or vehicles through calculation of tracing scores for venues and food products under investigation [20]. A premise linked to a confirmed case was weighted (assigned as 1 vs. 0 for holdings without confirmed cases). Tracing scores were calculated for all movements. Tracing scores are a value between 0 and 1 . Higher tracing scores are correlated with the probability that an animal moved on or off a holding and may help to identify a common contamination source.

\section{RESULTS}

\section{Epidemiological investigation of the outbreak associated with consumption of contaminated salad}

On 11 August 2015, four cases of STEC O157:H7 stx2a PT8 belonging to the same five SNP single linkage cluster $(18 \cdot 35 \cdot 380 \cdot 765 \cdot 1009 \cdot 1526 . \%)$ were identified. By 31 October 2015, the cluster comprised 47 cases (Fig. 1). Onset dates for confirmed and probable cases ranged from 29 July to 30th September 2015 (Fig. 2). None of the cases developed HUS, but 16 $(34 \%)$ were hospitalised and 34 cases $(72 \%)$ had blood in their stools. The majority of cases were over 18 years of age $(88 \%)$ and female $(69 \%)$. Cases were dispersed throughout England, and there was one case in South Wales (Fig. 2). The highest burden of cases was in the North West, South East and East of England (nine cases each).

An exposure frequency analysis conducted on SESQs revealed that the most common exposure reported by cases was consumption of prepacked salad $(38 / 47 ; 81 \%)$ and of these, $22(58 \%)$ had purchased their prepacked salad at the same national supermarket chain (retailer X). The case-case study included 36 cases and 78 non-outbreak controls from the NESSS database. Results of the final multivariable model are shown in Table 1. Consumption of prepacked salad from retailer $\mathrm{X}$ was identified as

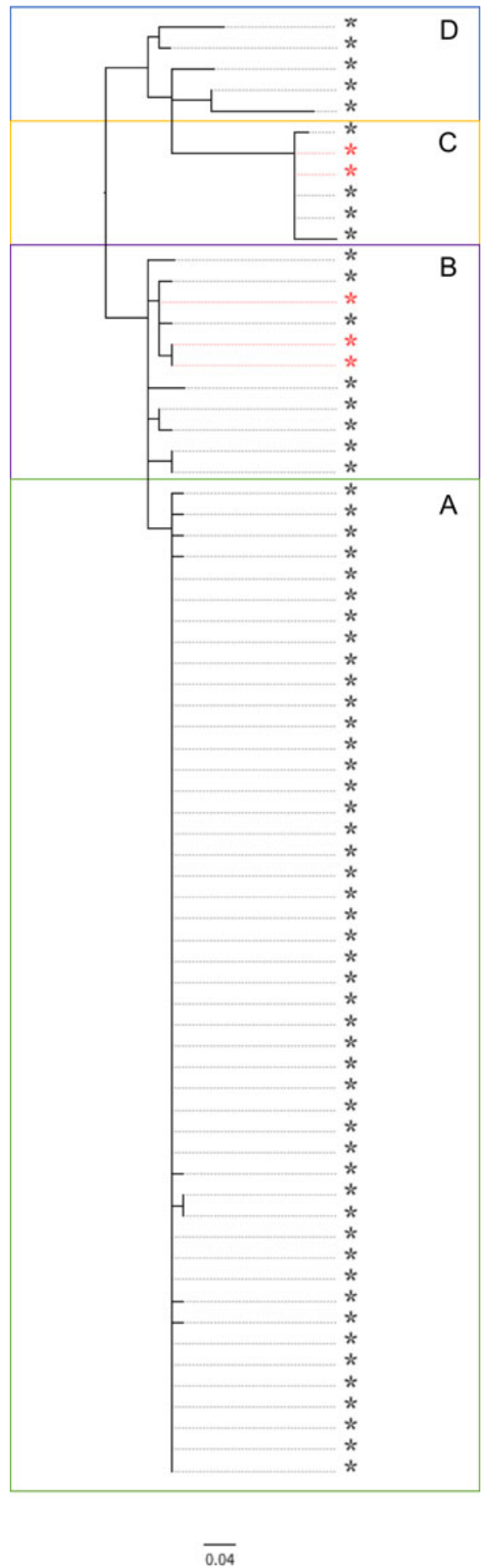

Fig. 1. Phylogenetic relationship between the isolates linked to the salad outbreak sub-cluster A (highlighted in green), and non-salad outbreak isolates (sub-clusters B, C and D) within the same $10 \mathrm{SNP}$ cluster. Sub-cluster B (highlighted pink) - contaminated lamb mince; sub-cluster C - contaminated lamb sausage (highlighted yellow); subcluster D (highlighted blue) - direct contact with sheep or lambs or their environment in the northwest of England. Food isolates are highlighted with a red asterisk. 


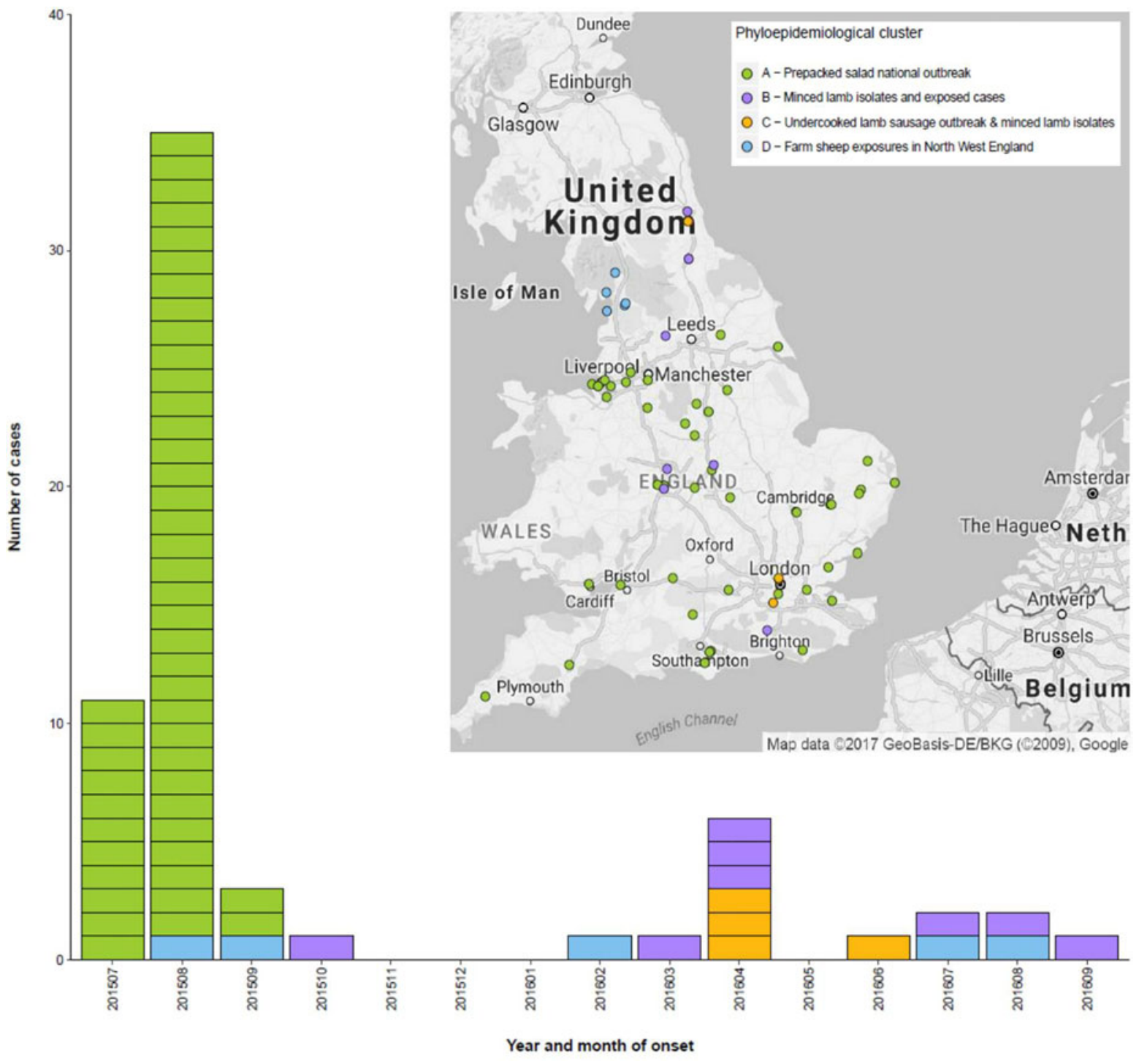

Fig. 2. Temporal and geographic distribution of cases within the $10 \mathrm{SNP}$ cluster incorporating the outbreak attributed to consumption of contaminated prepacked salad and sporadic cases detected following the outbreak, UK, July 2015 December 2016. Sub-cluster A (highlighted green) - salad outbreak; sub-cluster B (highlighted pink) - contaminated lamb mince; sub-cluster C - contaminated lamb sausage (highlighted yellow); sub-cluster D (highlighted blue) - direct contact with sheep or lambs or their environment in the northwest of England.

the primary exposure associated with the outbreak (OR 54, 95\% CI 11-247).

\section{Trace back and environmental investigations of the outbreak associated with consumption of contaminated salad}

A focused trawl was then carried out on the first 24 cases to obtain further details on the type of prepacked salad consumed. Two salad product lines (bistro salad and mild mixed leaf salad) were implicated. A trace back investigation revealed that both salads shared an ingredient (green multi-leaf lettuce) and were processed and packed by a single distributor in the North West of England. The distributor was supplied by five UK salad growers (Fig. 3). The five source farms were visited and inspected. A review of routine test results of the produce revealed that high indicator $E$. coli levels had been detected in packaged produce from one farm in August 2015 during the outbreak. In addition, three farms used untreated irrigation water from open ponds $(n=2)$ or rivers $(n=1)$. Potential risks, or actual evidence, of animal ingress (wildlife) was identified at all farms. Follow-up of all 
Table 1. Odds ratios for multivariable analysis of the outbreak associated with consumption of contaminated salad

\begin{tabular}{|c|c|c|c|c|c|c|}
\hline \multirow[b]{2}{*}{ Exposure } & \multicolumn{2}{|c|}{ Cases exposed* } & \multirow{2}{*}{$\begin{array}{l}\text { Odds ratio } \\
\text { (adjusted) }\end{array}$} & \multirow[b]{2}{*}{$P$-value } & \multicolumn{2}{|c|}{$95 \%$ confidence interval } \\
\hline & No. & $\%$ & & & Lower & Upper \\
\hline Prepacked salad from retailer X & 22 & 49 & 54 & $<0 \cdot 001$ & 11 & 247 \\
\hline Prepacked salad from other retailer & 12 & 27 & $5 \cdot 8$ & $<0.05$ & $1 \cdot 3$ & 26 \\
\hline Raw lamb & 6 & 13 & 10 & $<0 \cdot 05$ & $1 \cdot 2$ & 89 \\
\hline Fish & 27 & 60 & $2 \cdot 5$ & $0 \cdot 12$ & $0 \cdot 8$ & $7 \cdot 9$ \\
\hline Raw poultry & 16 & 36 & $1 \cdot 8$ & $0 \cdot 32$ & $0 \cdot 5$ & $6 \cdot 2$ \\
\hline Sex (male) & - & - & $1 \cdot 2$ & $0 \cdot 81$ & $0 \cdot 3$ & 4 \\
\hline Age & - & - & 1 & $0 \cdot 12$ & 0.9 & 1 \\
\hline
\end{tabular}

* Number and proportion of primary cases exposed were calculated from a total of 45 cases. Of these, 36 were included in the case-case study and compared to 78 controls to derive the odds ratios displayed above.

potential areas of sub-optimal practice was undertaken by local or national enforcement authorities.

\section{Microbiological examination of animal faecal specimens, food and environmental samples}

Sampling and microbiological testing of the packaged products were undertaken from a selection of supermarkets visited by cases. In addition, lettuce leaves, chlorine wash water and environmental swabs from cutting and conveying equipment at the processing plant were tested for STEC. STEC was not detected in any of the samples tested, and no episodes of gastrointestinal illness had been reported from workers at the processing plant during the period of interest. A total of 32 salad leaf samples and 13 irrigation water samples from the source farms were tested, and STEC was not detected in any of the samples.

\section{Phylogenetic relationships between isolates from outbreak and post-outbreak cases}

After the outbreak was declared over, analysis of the WGS data identified 22 additional isolates that fell within a 10 SNP single linkage cluster of the outbreak strain. Of these, 17 isolates were from symptomatic human cases, and five isolates were from food products made from lamb meat sent for routine testing to a commercial food microbiology laboratory from a national retailer. Of the 17 human cases, three reported onset of symptoms between August and October 2015, and 14 reported onset of symptoms between March 2016 and September 2016 (Fig. 2).

Using a combination of epidemiological data and phylogenetic analysis, the outbreak and post-outbreak cases belonging to this 10 SNP single linkage cluster
(SNP address: $18 \cdot 35 \cdot 397 \cdot 765 \cdot 1009 . \%$ ) were categorised into four sub-clusters (Fig. 1). Sub-cluster A (highlighted in green in Figs 1 and 2) comprised 47 cases associated with the salad outbreak in 2015 (SNP variation $0-1$ ). Sub-cluster B contained 11 isolates (SNP variation $0-4$ ) received at the reference laboratory between October 2015 and September 2016. Sub-cluster B (highlighted in pink in Figs 1 and 2) included three isolates detected in lamb meat and eight geographically dispersed cases (Fig. 2), suggesting a nationally distributed food product was associated with infection. Seven of the eight cases reported consumption of sausages and/or burgers prior to onset of symptoms, of which four specifically reported consumption of food products made from lamb meat. Sub-clusters A and B were separated by a minimum distance of three SNPs (Fig. 1) and were estimated to share a common ancestor approximately $1 \cdot 6$ years ago, in early 2015 (95\% HPD 1.5-2.9). Subcluster C (highlighted in yellow in Figs 1 and 2) comprised six isolates; four human cases linked to an outbreak caused by the consumption of undercooked lamb sausages at the same venue and two isolates from food products made from lamb meat (SNP variation 0-4). Sub-cluster D (highlighted in blue in Figs 1 and 2) was ancestral to the other sub-clusters and comprised five cases all resident in the northwest of England (SNP variation 8-25) (Fig. 2). The mostrecent common ancestor of four sub-clades was estimated to the middle of 2014 (95\% HPD 1.8-5.3).

\section{Epidemiological investigation of the links between cases in the $10 \mathrm{SNP}$ cluster}

Links between the sub-clusters were investigated in order to identify a common epidemiological source 


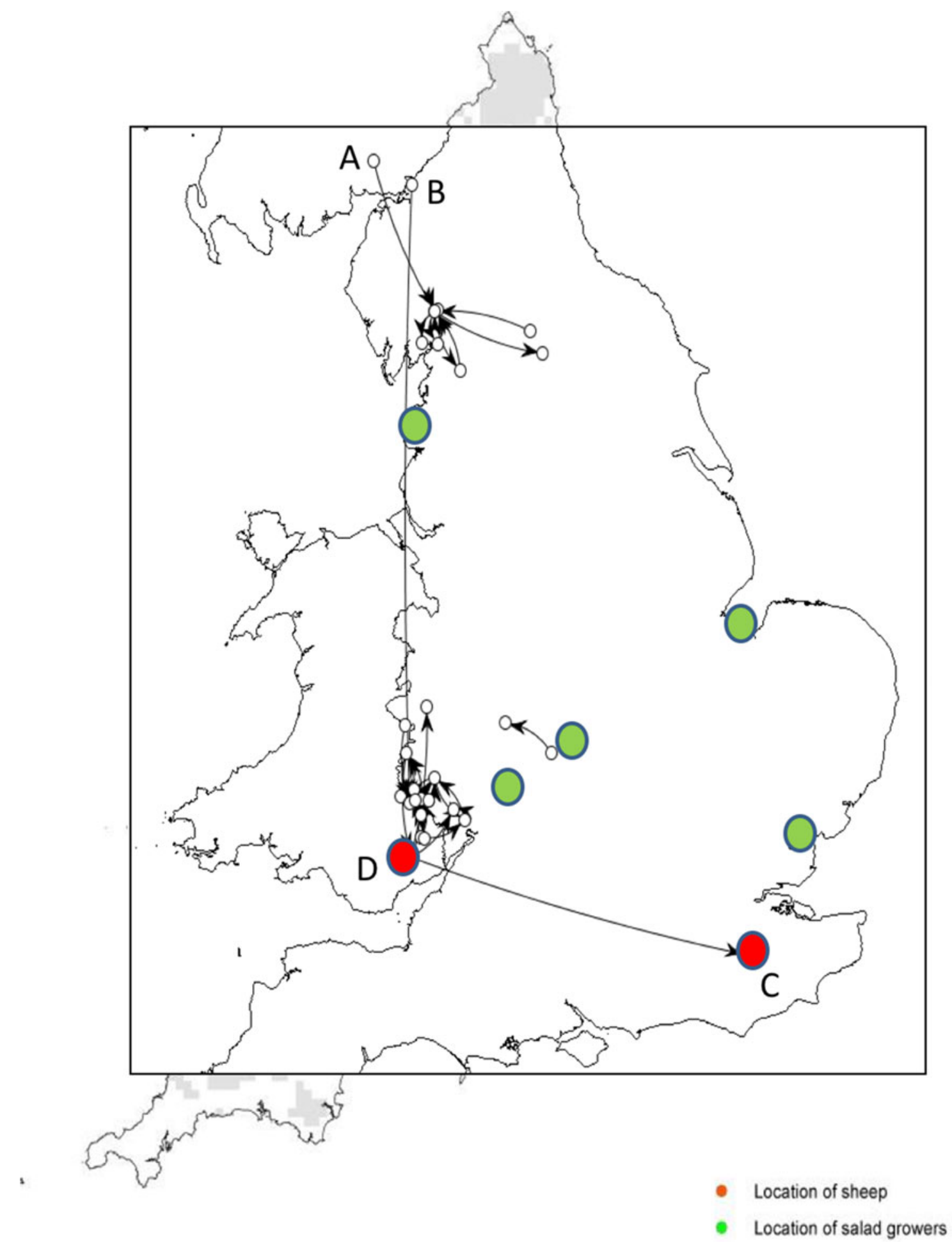

Fig. 3. Analysis of sheep movement data showed that lamb flocks sourced for the contaminated lamb meat products at farms $\mathrm{C}$ and D, and a network of lamb and sheep movement in the northwest of England, originated from a single $20 \mathrm{~km}$ radius area incorporating farms A and B. Green circles represent the location of the salad growers implicated in the outbreak sub-cluster A.

and clarify transmission routes. A second case-case study comprising cases detected from 2016 onwards that were not part of the outbreak associated with the consumption of contaminated salad leaves, revealed that ovine exposures, including direct contact with sheep or lambs, and/or the handling and consumption of lamb meat (particularly minced meat products), were most strongly associated with illness 
Table 2. Odds ratios for multivariable analysis of non-outbreak cases phylogenetically linked (10 SNP single linkage cluster) to an outbreak caused by consumption of contaminated salad to test an ovine exposure hypothesis

\begin{tabular}{|c|c|c|c|c|c|c|}
\hline \multirow[b]{2}{*}{ Exposure } & \multicolumn{2}{|c|}{ Cases exposed* } & \multirow{2}{*}{$\begin{array}{l}\text { Odds ratio } \\
\text { (adjusted) }\end{array}$} & \multirow[b]{2}{*}{$P$-value } & \multicolumn{2}{|c|}{$95 \%$ confidence interval } \\
\hline & No. & $\%$ & & & Lower & Upper \\
\hline Ovine (meat or animal contact) & 12 & 75 & $8 \cdot 24$ & $0 \cdot 008$ & $1 \cdot 55$ & $39 \cdot 74$ \\
\hline Sandwiches & 9 & 56 & $2 \cdot 59$ & $0 \cdot 071$ & $0 \cdot 92$ & $7 \cdot 52$ \\
\hline Prepacked salad & 8 & 50 & $1 \cdot 90$ & $0 \cdot 24$ & $0 \cdot 64$ & $5 \cdot 65$ \\
\hline Cured meat & 8 & 50 & $1 \cdot 84$ & $0 \cdot 25$ & $0 \cdot 64$ & $5 \cdot 23$ \\
\hline Raw vegetables & 13 & 81 & $1 \cdot 92$ & $0 \cdot 33$ & $0 \cdot 57$ & $8 \cdot 80$ \\
\hline Lamb meat & 9 & 56 & $1 \cdot 01$ & $0 \cdot 99$ & $0 \cdot 27$ & $4 \cdot 92$ \\
\hline Sex (male) & - & - & $0 \cdot 93$ & $0 \cdot 89$ & $0 \cdot 32$ & $2 \cdot 59$ \\
\hline Age (years) & - & - & $1 \cdot 01$ & $0 \cdot 40$ & $0 \cdot 99$ & $1 \cdot 03$ \\
\hline
\end{tabular}

* Number and proportion of primary cases exposed were calculated from a total of 18 cases. Of these, 16 were included in the case-case study and compared to 1919 controls to derive the odds ratios displayed above.

$(n=16$; aOR $8 \cdot 24 ; 95 \%$ CI $1 \cdot 55-39 \cdot 74)$. Other exposures that met the criteria for inclusion in the final model (consumption of sandwiches, prepacked salad, cured meat and raw vegetables) were not significant (Table 2).

Details of the movements of sheep and lambs to and from farms and other locations were supplied for a total of 30 sites associated with lambs being held prior to slaughter or cases having direct or indirect contact with lambs and/or sheep comprising a total of 1238 sheep in 89 independent movements between sites. The analysis revealed two distinct networks of sheep movements originating from two farms (farms A and B) within $20 \mathrm{~km}$ of each other on the Scottish-English border (Fig. 3). The network in the northwest of England developed from a single movement of 22 sheep from farm A in Scotland. The second network in the Welsh border region, developed from three independent movements of 138 sheep from farm B in Cumbria. Each movement contained 50, 38 and 50 animals, respectively.

Product trace back and analysis of sheep movement data showed that the lambs associated with the contaminated lamb sausage were sourced from farm $\mathrm{C}$ in the southeast of England. The lambs from farm $\mathrm{C}$ were directly linked to farm D on the Welsh-English border. Farm D was the source of the lambs associated with the contaminated mince (Fig. 3).

\section{DISCUSSION}

In England over the last 10 years, there has been an increase in reports of STEC O157:H7 outbreaks associated with raw vegetables or salad leaves [4-7].
Investigations of these outbreaks can be challenging, as they are often small in case numbers, geographically dispersed and the contaminated product is often a minor component of a meal that people may not remember. In addition, the source of the leaves used and the composition of bagged salad products changes frequently and distribution chains may be complex, making trace back to specific farms extremely difficult. Furthermore, salad produce has a short shelf-life and may contain very low levels of bacteria that are not detectable by microbiological testing [8]. Routine microbiological monitoring performed by the food business operator includes tests for indicator bacteria used to detect and estimate the level of faecal contamination, but are not specific for STEC O157:H7.

Increased awareness of consumption of contaminated salad leaves as a potential source of STEC O157:H7 infection has improved the quality of food history data from cases recorded on the SESQ in England. In this outbreak, pre-packed salad was quickly identified as the most likely source in the initial phase of the investigation, and this was confirmed by the case-case study. This was the first time that SESQs had been used for an analytical study. This approach reduces recall error, because questioning is completed relatively quickly after initial presentation, and reduces recall and interviewer bias because at the time of interview, the case would not have been classified as an outbreak case. However, using the SESQs for a case-case study does risk overmatching and does not allow the questions to be modified to precisely match the hypothesis, both of which may reduce study power to detect a real association. 
During this study, despite the risk of a high salad exposure in the control group, the outbreak strain cases were 54 times more likely to have consumed prepacked salad (95\% CI 11-247). Detailed follow-up of the cases confirmed the hypothesis and directed the trace back investigation to focus on green multi-leaf lettuce packed by a single supplier who received produce from five farms.

This investigation was the first time that data from ARAMS has been used to investigate epidemiological links between phylogenetically related isolates of STEC O157:H7. The analysis was limited because at the time of this investigation the ARAMS did not hold a complete record of all sheep movements that had occurred during the time frame of interest. In particular, data were not available for the sheep holdings in the vicinity of the growers irrigating salad crops with untreated water. Ultimately, a definitive link could not be established between sub-cluster A (cases associated with the consumption of contaminated salad leaves) and the other three sub-clusters. However, a direct link was established between the isolates in sub-clusters B (cases associated with the consumption of contaminated lamb mince) and $\mathrm{C}$ (cases associated with the consumption of contaminated lamb sausage) via the movements of lambs between farm $\mathrm{C}$ in the southeast of England and farm D on the Welsh borders. A potential geographical link was identified between sub-clusters B (cases associated with the consumption of contaminated lamb mince) and D (cases associated with direct contact with lambs and/or sheep and their environment) as their associated sheep movement networks could be traced back to two farms $20 \mathrm{~km}$ apart.

Animal faeces may be distributed over wide areas by stream and river systems following heavy rainfall. More timely and intensive sampling of the location of the farms where the green multi-leaf lettuce was produced, including the irrigation water and cattle and/or sheep grazing upstream of the irrigation point, may have facilitated the detection of the outbreak strain in these animals, or in their environment [21]. In a previous outbreak associated with consumption of contaminated watercress, although not detected in the watercress, STEC O157:H7 was successfully detected in water used to irrigate the crop on the farm [8]. During an investigation of an outbreak of STEC O157:H7 caused by contaminated raw drinking milk, the causative organism was not detected in the milk but was isolated from faecal pats from the cows producing the milk [9].
Microbiological testing in response to the outbreak failed to identify the pathogen in food collected during the investigation. More appropriate testing using PCR assays targeting the stx genes may enable the identification of contaminated of ready-to-eat vegetables, including salad vegetables, before and after they enter the food chain. Salad vegetables are considered ready to eat food products. Washing during and after processing is largely ineffective in removing bacteria, particularly those that are internalised, the most important food safety controls are those applied in the field [22]. In England, salad production is highly localised to small parts of the country, and some areas are geographically close to areas of high animal density, particularly in the northwest and southwest of the country. The likelihood of direct or indirect contamination of ready to eat salad crops (e.g. from birds, wildlife or contaminated watercourses) from the animal reservoir is likely to be higher in these areas. Farms growing salad vegetables are deemed primary producers. The legal requirements for hygiene in primary production are laid out in EC Regulation 852/2004 which applies the principles of good agricultural practice and good hygiene practice but are not as stringent as those required for food processing and production. However, business must be able to demonstrate that their operations are managed in a way that control food safety risks, including those associated with the use of water, and primary growers must use potable or clean water to prevent contamination.

During this study, the potential for identifying the geographical origin of an outbreak strain by analysing epidemiological data associated with cases in phylogenetically related sub-clusters was explored. Although, a robust link between the outbreak subcluster (A) and the other three ovine associated subclusters B, C and D (linked to consumption of lamb mince, consumption of lamb sausage and direct contact with sheep or lambs, or their environment, respectively) was not confirmed, epidemiological links were established between three ovine sub-clusters by mapping the recent movement of sheep and lambs across the UK. Given the close phylogenetic relationship between the outbreak strain and the isolates from cases with ovine exposures, it is plausible that ovine faeces may have contaminated the salad leaves via untreated irrigation water or run-off from fields nearby. Timely and targeted veterinary and environmental sampling should be considered during outbreaks of STEC, particularly where ready to eat vegetables are implicated. 


\section{SUPPLEMENTARY MATERIAL}

The supplementary material for this article can be found at https://doi.org/10.1017/S0950268817002874.

\section{ACKNOWLEDGEMENTS}

The authors would like to thank Neil Perry, Vivienne do Nascimento and Marie Chattaway at GBRU, Kate James from PHE WM Centre and colleagues at PHE Food, Water and Environmental Microbiology Laboratories and Preston, Porton and Birmingham. The authors would also like to acknowledge everyone who was part of the Outbreak Control Team including Drazenka Tubin-Delic, Jonathan Lighthill and Joanne Edge the Food Standards Agency, Paul Homes, Alan Wright and Charlotte Featherstone at the Animal and Plant Health Agency and Eamon Rodgers at Worcestershire Regulatory Services County Council. This work was supported by the National Institute for Health Research Health Protection Research Unit in Gastrointestinal Infections. Jeremy Hawker, Richard Elson, Paul Cleary, Tim Dallman and Tom Inns are affiliated to the National Institute for Health Research Health Protection Research Unit (NIHR HPRU) in Gastrointestinal Infections at University of Liverpool in partnership with PHE, in collaboration with University of East Anglia, University of Oxford and the Institute of Food Research. The views expressed are those of the author(s) and not necessarily those of the NHS, the NIHR, the Department of Health or PHE.

\section{REFERENCES}

1. Adams NL, et al. Shiga toxin-producing Escherichia coli O157, England and Wales, 1983-2012. Emerging Infectious Diseases 2016; 22: 590-597.

2. Chapman PA. Sources of Escherichia coli O157 and experiences over the past 15 years in Sheffield, UK. Symposium Series Society Applied Microbiology 2000; 29: $51 \mathrm{~S}-60 \mathrm{~S}$.

3. Byrne L, et al. The epidemiology, microbiology and clinical impact of Shiga toxin-producing Escherichia coli in England, 2009-2012. Epidemiology and Infection 2015; 143: 3475-3487.

4. Launders N, et al. Outbreak of Shiga toxin-producing Escherichia coli $\mathrm{O} 157$ associated with consumption of watercress, United Kingdom, August to September 2013. Euroeurosurveillance 2013; 18: 44.

5. Byrne L, et al. Epidemiological and microbiological investigation of an outbreak of severe disease from Shiga toxin-producing Escherichia coli O157 infection associated with consumption of a slaw garnish. Journal of Food Protection 2016; 79: 1161-1168.
6. Sinclair C, et al. Investigation of a national outbreak of STEC O157 using online consumer panel control methods: Great Britain, October 2014. Epidemiology and Infection 2016; 14: 1-8.

7. Launders $\mathbf{N}$, et al. A large Great Britain-wide outbreak of STEC O157 phage type 8 linked to handling of raw leeks and potatoes. Epidemiology and Infectious 2016; 144: $171-181$.

8. Jenkins C, et al. Public health investigation of two outbreaks of Shiga toxin-producing Escherichia coli O157 associated with consumption of watercress. Applied and Environmental Microbiology 2015; 81: 3946-3952.

9. Butcher $\mathbf{H}$, et al. Whole genome sequencing improved case ascertainment in an outbreak of Shiga toxin-producing Escherichia coli $\mathrm{O} 157$ associated with raw drinking milk. Epidemiology and Infection 2016; 144: 2812-2823.

10. Rowell S, et al. An outbreak of Shiga toxin-producing Escherichia coli serogroup O157 linked to a lamb-feeding event. Epidemiology and Infection 2016; 144: 2494-2500.

11. Joensen KG, et al. Rapid and easy in silico serotyping of Escherichia coli isolates by use of whole-genome sequencing data. Journal of Clinical Microbiology 2015; 53: 2410-2426.

12. Li H, Durbin R. Fast and accurate long-read alignment with Burrows-Wheeler transform. Bioinformatics 2010; 26: 589-595.

13. McKenna A, et al. The genome analysis toolkit: a MapReduce framework for analyzing next-generation DNA sequencing data. Genome Research 2010; 20: 1297-1303.

14. Stamatakis A. RAxML version 8: a tool for phylogenetic analysis and post-analysis of large phylogenies. Bioinformatics 2014; 30: 1312-1313.

15. Dallman TJ, et al. Whole-genome sequencing for national surveillance of Shiga toxin-producing Escherichia coli O157. Clinical Infectious Disease 2015; 61: 305-312.

16. Dallman TJ, et al. SnapperDB: a database solution for routine sequencing analysis of bacterial isolates. bioRxiv 2017; 189118. https://www.biorxiv.org/content/early/2017/ 09/15/189118.

17. Ashton PM, et al. Insight into Shiga toxin genes encoded by Escherichia coli $\mathrm{O} 157$ from whole genome sequencing. PeerJ 2015; 3: e739.

18. Dallman TJ, et al. Applying phylogenomics to understand the emergence of Shiga-toxin-producing Escherichia coli $\mathrm{O} 157: \mathrm{H} 7$ strains causing severe human disease in the UK. Microbial Genomics 2015; 1: e000029.

19. Feinerer I, et al. Text mining infrastructure in R. Journal of Statistical Software 2008; 25: 1-54.

20. Weiser AA, et al. Food-Chain-Lab: a trace-back and trace-forward tool developed and applied during foodborne disease outbreak investigations in Germany and Europe. PLoS ONE 2016; 11: e0151977.

21. Söderström A, et al. A large Escherichia coli $\mathrm{O} 157$ outbreak in Sweden associated with locally produced lettuce. Foodborne Pathogens and Disease 2008; 5: 339-349.

22. Shaw RK, et al. Enterohemorrhagic Escherichia coli exploits EspA filaments for attachment to salad leaves. Applied and Environmental Microbiology 2008; 74: 2908-2914. 